\author{
Military Technical College \\ Kobry El-kobbah, \\ Cairo, Egypt
}

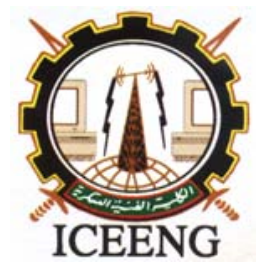

\section{$5^{\text {th }}$ International Conference on Electrical Engineering}

ICEENG 2006

\title{
FIELD ANALYSIS AND EQUIVALENT CIRCUIT PARAMETERS OF DISC-ROTOR INDUCTION MOTOR
}

\author{
M.k. Ahmed A.B.Kotb M.A. Zaher \\ Al-Azhar University, Faculty of Engineering, Cairo, Egypt.
}

\begin{abstract}
$\underline{\text { Abstract }}$
The disc-rotor induction motors come in a variety of structures such as double-sided stator, single-sided stator with iron backing rotor, and single-sided stator without iron backing rotor. This paper presents the field analysis of the disc-rotor induction motor using two-dimension analysis of magnetic field. The computational treatments are based on the solution of field equations written down in cylindrical coordinates. This analysis is carried out using the vector potential, which is shown to be readily adaptable to the evaluation of the air-gap impedance for different disc motor constructions. The magnetic vector potential enables the effect of physical air-gap and finite permeability and conductivity of a number of machine regions to be taken into account. Using this new technique, the complex expression of the impedance can be rearranged to predict the individual equivalent circuit parameters.
\end{abstract}

\section{List of Symbols:}

\begin{tabular}{|c|c|c|c|}
\hline $\mathrm{A}_{\mathrm{s}}$ & $=$ stator electric loading & $\mathrm{R}_{\mathrm{i}}, \mathrm{R}_{\mathrm{o}}$ & $=$ inner and outer rotor radius, \\
\hline$a_{\varphi}, a_{\mathrm{r}}$ & $=$ unit vectors & $\mathrm{R}_{\mathrm{s}}, \mathrm{R}_{\mathrm{r}}$ & $=$ stator and rotor resistance \\
\hline a & $=$ wave length ratio, & $\mathrm{S}$ & $=$ slip \\
\hline $\mathrm{B}_{\mathrm{z}, \mathrm{B}_{\varphi}}$ & $=$ flux density components, & $t_{c}$ & $=$ rotor thickness, \\
\hline $\mathrm{E}$ & $=$ electric field intensity & $\mathrm{t}$ & $=$ time \\
\hline $\mathrm{f}$ & $=$ frequency & V & $=$ magnetic vector potential, \\
\hline g & $=$ air-gap length, & $\mathrm{X}_{\mathrm{m}}$ & $=$ magnetizing reactance \\
\hline $\mathrm{H}$ & $=$ magnetic field intensity, & $\mathrm{X}_{\mathrm{s}}, \mathrm{X}_{\mathrm{r}}$ & $=$ stator and rotor reactances \\
\hline $\mathrm{J}_{\mathrm{r}}$ & $=$ rotor current density & $\mathrm{V}$ & $=$ linear velocity \\
\hline $\mathrm{p}$ & $=$ number of pole pairs, & $\mathrm{z}, \varphi, \mathrm{r}$ & $=$ coordinates \\
\hline$p, q, k$ & $=$ complex constants & $\sigma$ & $=$ disc-rotor conductivity \\
\hline$r_{i}, r_{o}$ & $=$ inner and outer stator radius, & $\omega$ & $=$ angular frequency. \\
\hline$Z_{g}$ & $=$ air-gap impedance & $v$ & $=$ motor relative speed \\
\hline
\end{tabular}

\section{Introduction}

A disc motor with an axial air-gap and a flat homogeneous rotor shows some performance characteristics, which are superior to conventional motor. One of the principal features of this motor is the small inertia that makes it a suitable choice for 
servo and high-speed applications[1]. The disc-rotor induction machine promises high utilization of the active materials and thus favorable power density [2]. The simplicity in establishing the current distribution in the disc-rotor by means of induction from the excited stator provides robust and cheep construction. The excitation system, which transfers power to the secondary disc, consists of polyphase windings inserted into radially directed slots[4].

S.E Abdollahi, M. Mirsalim, M. Mirzayee and A. Vahedi [1,3] derived a mathematical model for disc-rotor induction motor, that analysis includes numerical and analytical methods using Maple software. A.B.Kotp suggested an interesting method for control of two-phase disc-rotor induction motor [2]. Here, a method for motor parameters prediction is presented. Generally, electrical machine designers prefer to use mathematical models that respond in terms of the physical phenomena of the machine. This enables the designer to envision what is going on inside a machine, giving him guidance on how to vary the machine dimensions and winding types in order to achieve the required performance. The analytical approach described herein, leads to a mathematical model, which is also a practical design tool.

In this paper, three disc motor constructions are proposed and their models are considered taking into account the rotor thickness also can be considerably simplified by assuming thin rotor with finite surface conductivity. The construction and basic elements of a disc-rotor induction motor are shown schematically in Fig.1. The airgap impedances and the equivalent circuit parameters of the disc motors are determined by analysis, which provides a comprehensive technique for design and for assessment of performance.

The field analysis is carried out using Maxwell's equations in cylindrical coordinates [5] are derived and solved for the three regions of the model. The magnetic field is assumed to remain constant along the mechanical air-gap between the two stators, while the current density in the disc rotor is allowed to have radial and tangential components. The field distribution and rotor current density are given for different design parameters of the disc rotor induction motor.

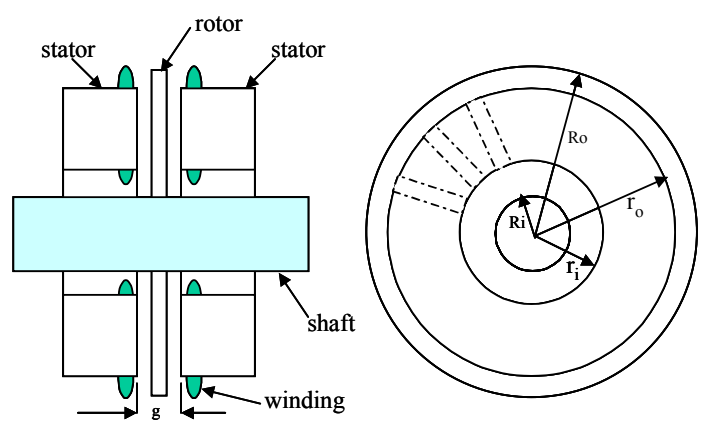

Fig. 1 Disc-rotor induction motor construction.

Deferent constructions of disc motor are shown in Fig. 2, where (a) is a double-sided stator model, (b) is single sided stator with iron backing rotor model, and (c) is a single sided stator without iron backing rotor model. 


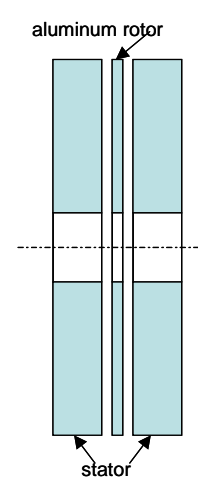

(a)

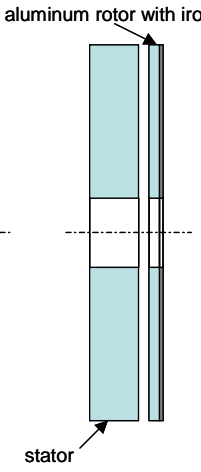

(b)

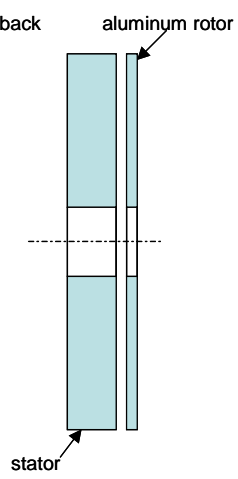

(c)

Fig. 2 Disc motor models for three different design data, (a) Two stator model, (b) One stator with iron backing rotor, (c) One stator without iron backing rotor.

\section{Mathematical Model}

It is evident that the knowledge of the exciting stator electric loading is necessary for the electromagnetic field calculations. The stator electric loading $A_{s}(A / m)$ should be expressed in a form suitable for the solution of the field equations [2]. The slotted stator is replaced by a smooth iron core carrying a continuous current sheet representing the stator electric loading [6]. In general the excitation system which produces the rotating magnetic field consists of one-or two-phase windings embedded in radially directed slots.

The computation coordinates are depicted in Fig.3, and the following assumptions are made for modeling:

1) The axial component $B_{z}$ and tangential component $B_{\varphi}$ of the flux densities, are considered.

2) The variation of field intensity and currents along the z-axis are zero.

3) The relative permeability of stator ferromagnetic parts is considered to be infinity The sum of the two stator electric loadings can be expressed in complex form as:

$$
\left.A_{S}(\varphi, t)=\Re A_{S} \cdot e^{j(\omega t-p \varphi)}\right\}
$$

The magnetic field analysis in cylindrical coordinates is performed starting with the Maxwell's field equations and based on the three- regions model shown in Fig. 3. The three regions of the model are defined as follows:

region 1: lower air-gap $0 \leq \mathrm{z} \leq \mathrm{g}_{1}$, region 2: disc-rotor $\mathrm{g}_{1} \leq \mathrm{z} \leq \mathrm{g}_{1}+\mathrm{t}_{\mathrm{c}}$, region 3: upper air-gap $\mathrm{g}_{1}+\mathrm{t}_{\mathrm{c}} \leq \mathrm{z} \leq \mathrm{g}_{1}+\mathrm{t}_{\mathrm{c}}+\mathrm{g}_{2}$. 


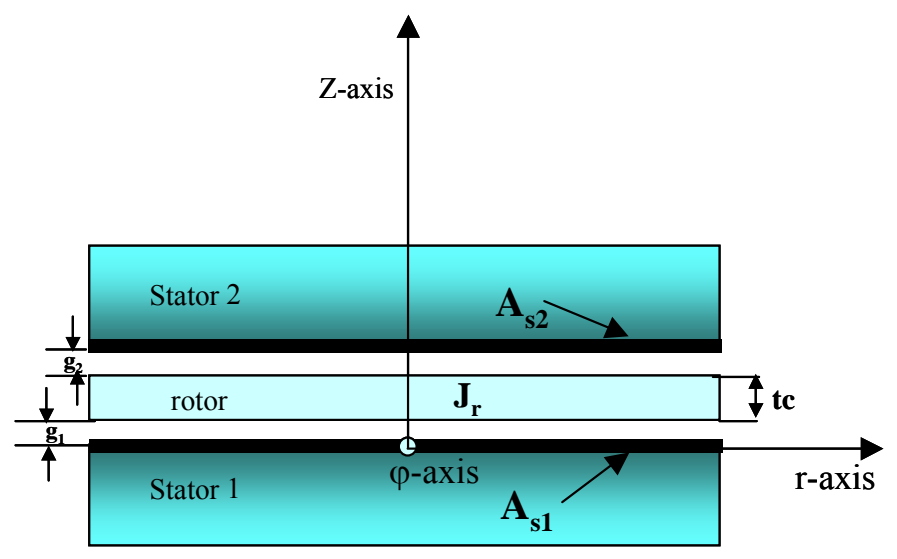

Fig. 3 Model of the disc-rotor induction motor.

With respect to the stationary cylindrical coordinates fixed to the stator, Maxwell's field equations for a rotating medium are given by: -

$\mathrm{CurlH}=\mathrm{J}$,

$\operatorname{Curl} \mathrm{E}=\frac{-\partial B}{\partial t}+\operatorname{Curl}(\mathrm{v} \times \mathrm{B})$

Where the linear speed $\mathrm{v}$ of the conducting disc-rotor is taken along the $\varphi$ direction. The magnetic vector potential $\mathrm{V}$ is defined by:

$\mathrm{B}=\mathrm{Curl} \mathrm{V}$

Neglecting the end effects in both stator and rotor, the vector potential will have only a radial $r$-component as:

$V=V_{r} a_{r}$

The magnetic vector potential must be similar to the exciting stator electric loading i.e. it is to be represented in the form[2]:

$V_{r}(t, \varphi, z)=\mathfrak{R} e\left\{V_{r}(z) \cdot e^{j(\omega t-p \varphi)}\right\}$

Thus, we obtain the differential equations describing the vector potential in the different regions of the model:

$$
\begin{aligned}
& \frac{\partial^{2} V_{r 1}}{\partial z^{2}}-a^{2} \cdot V_{r 1}=0 \\
& \frac{\partial^{2} V_{r 2}}{\partial z^{2}}-k^{2} \cdot V_{r 2}=0 \\
& \frac{\partial^{2} V_{r 3}}{\partial z^{2}}-a^{2} \cdot V_{r 3}=0
\end{aligned}
$$


Where

$$
a=\frac{2 p}{R_{o}+R_{i}}, k=\sqrt{a^{2}-j \omega \sigma \mu_{0} s}
$$

The solutions of the above differential equations for the different regions of the model are:

$$
\begin{aligned}
& V_{r 1}=p_{1} \cdot e^{a z}+q_{1} \cdot e^{-a z} \\
& V_{r 2}=p_{2} \cdot e^{k z}+q_{2} \cdot e^{-k z} \\
& V_{r 3}=p_{3} \cdot e^{a z}+q_{3} \cdot e^{-a z}
\end{aligned}
$$

The unknown integration constants $p$ and $q$ are to be evaluated by applying the following boundary conditions :

1) Across each integration boundary, the $\varphi$-component of the magnetic field intensity $\mathrm{H}_{\varphi}$ is continuous. Introducing the exciting stator electric loading at the air-gap stator boundary can modify this condition:

$H_{\varphi}=\frac{1}{\mu} \frac{\partial V_{r}}{\partial z}$

and we have, at the boundary between regions $n$ and $n+1$

$$
\frac{1}{\mu_{n}} \frac{\partial V_{r n}}{\partial z}=\frac{1}{\mu_{n+1}} \frac{\partial V_{r n+1}}{\partial z}
$$

At lower air-gap boundary, introducing the exciting stator electric loading modifies the condition:

$$
\left.\frac{1}{\mu_{o}} \frac{\partial V_{r 1}}{\partial z}\right|_{z=0}=A_{s}
$$

Since $\mu$ tends to infinity. By applying Eq.(13) at the upper air-gap boundary yields:

$$
\left.\frac{1}{\mu_{o}} \frac{\partial V_{r 3}}{\partial z}\right|_{z=2 g+t_{c}}=0
$$

The same condition is to be applied at the lower air-gap and upper air-gap boundaries yielding:

$$
\begin{aligned}
& \left.\frac{1}{\mu_{o}} \frac{\partial V_{r 1}}{\partial z}\right|_{z=g}=\left.\frac{1}{\mu_{o}} \frac{\partial V_{r 2}}{\partial z}\right|_{z=g} \\
& \left.\frac{1}{\mu_{o}} \frac{\partial V_{r 2}}{\partial z}\right|_{z=g+t_{c}}=\left.\frac{1}{\mu_{o}} \frac{\partial V_{r 3}}{\partial z}\right|_{z=g+t_{c}}
\end{aligned}
$$


2) Across each integration boundary, the z-component of the flux density B, is continuous, which implies the continuity of the vector potential.

The flux density is given by :

$B_{z}=-\frac{\partial V_{r}}{\partial z}$

Which implies the continuity of the vector potential, so that

$V_{z n}=V_{z n+1}$

At the lower air-gap boundary the condition becomes:

$\left.V_{z 1}\right|_{z=g}=\left.V_{z 2}\right|_{z=g}$

By applying the condition at the upper air-gap boundary:

$\left.V_{z 2}\right|_{z=g+t_{c}}=\left.V_{z 3}\right|_{z=g+t_{c}}$

These condition are sufficiently to determine completely the integration constants $p$ and $q$, and hence the vector potential for all regions and flux density distribution and rotor current density can be obtained.

Application of the above boundary conditions to the separating surfaces at:

$\mathrm{z}=0, \mathrm{z}=\mathrm{g}_{1}, \mathrm{z}=\mathrm{g}_{1}+\mathrm{t}_{\mathrm{c}}$ and $\mathrm{z}=\mathrm{g}_{1}+\mathrm{t}_{\mathrm{c}}+\mathrm{g}_{2}, \mathrm{~g}_{1}=\mathrm{g}_{2}=\mathrm{g}$ and Eqs.(11)- (23) gives a set of simultaneous equations.

The solution of these equations gives the six integration constants $p$ and $q$ for the three regions of the model.

Substituting of the resulting values of $p$ and $q$ into equation (11)-(13) gives the vector potential for the three regions, and hence the flux density of the deferent machine regions as following:

$$
\begin{aligned}
& B_{z 1}=j a\left(p_{1} \cdot e^{a z}-q_{1} \cdot e^{-a z}\right) \\
& B_{\varphi 1}=a\left(p_{1} \cdot e^{a z}+q_{1} \cdot e^{-a z}\right) \\
& B_{z 2}=j a\left(p_{2} \cdot e^{k z}-q_{2} \cdot e^{-k z}\right) \\
& B_{\varphi 2}=a\left(p_{2} \cdot e^{k z}+q_{2} \cdot e^{-k z}\right) \\
& B_{z 3}=j a\left(p_{3} \cdot e^{a z}-q_{3} \cdot e^{-a z}\right) \\
& B_{\varphi 3}=a\left(p_{3} \cdot e^{a z}+q_{3} \cdot e^{-a z}\right)
\end{aligned}
$$

The air-gap flux density components are normalized by its no-load value $\mathrm{B}_{\mathrm{o}}$ hence:

$$
B_{o}=\frac{\mu_{o} A_{s}}{a\left(2 g+t_{c}\right)}
$$

According to the model the disc-rotor current density flows in the radial direction only, and its complex amplitude is then: 


$$
J_{r}=j w \cdot \sigma \cdot s \cdot\left(p_{2} \cdot e^{k z}+q_{2} \cdot e^{-k z}\right)
$$

The rotor current density components are normalized by the theoretical quantity $\mathrm{J}_{\mathrm{o}}$.

$$
J_{o}=\frac{A_{s}}{t_{c}}
$$

Now the distribution of the air-gap flux density and disc-rotor current density can be easily calculated, and plotted through the different machine regions for the three models. These calculations are carried out using the machine design data. In Fig.4 $(\mathrm{a}, \mathrm{b})$ the normalized axial $\mathrm{B}_{\mathrm{z}}$ and tangential $\mathrm{B}_{\varphi}$ flux density components are plotted against the axial air-gap length of the machine at constant speed for the three models.

It is clear from Fig (4a), that the axial flux components are constant allover the definition regions $\left(0 \leq \mathrm{z} \leq \mathrm{g}_{1}, \mathrm{~g}_{1} \leq \mathrm{z} \leq \mathrm{g}_{1}+\mathrm{t}_{\mathrm{c}}, \mathrm{g}_{1}+\mathrm{t}_{\mathrm{c}} \leq \mathrm{z} \leq \mathrm{g}_{1}+\mathrm{t}_{\mathrm{c}}+\mathrm{g}_{2}\right)$. The model (b), in which the motor has one stator with iron backing rotor, the axial flux density has the largest value than the models (a) and (c). This attributed to the shortest air-gap length. The model (c) has infinite air-gap length, therefore the magnetic field intensity is low, and hence the axial flux has low value because it is direct proportional to magnetic field intensity.

Fig.4 (b) shows the air-gap tangential flux density components for the three models at constant speed. The values of tangential flux density are decreased over all the air-gap bounders. The tangential component reduced to zero at end of the air-gap length and this is due to the infinite permeability of the second stator (model a) or iron backing model (b). For model (c), the tangential component reduced to zero at infinity. The axial distribution of the tangential air-gap flux density is consistent with the boundary condition between the tangential field components at both sides of disc- rotor induction motor.

The effect of the rotor speed on the axial and tangential flux distribution of the air-gap flux density components for model (a) can be shown in Fig. (5) and Fig (6) respectively, in Fig. (5) the axial flux component is direct proportional to the speed and has a constant value over all the air-gap bounders. In Fig. (6), the tangential flux component decreases with increasing of the speed before the middle of the rotor disc, but the situation is reversed after the middle of the rotor disc and reached to zero.

Fig. (7) shows the predicted values of the rotor current density distribution normalized by theoretical quantity of the rotor current density $\mathrm{J}_{\mathrm{o}}$ for varies motor construction models at constant speed. The rotor current density is consistent with the disc rotor boundary, and has a higher value for the model (b). Fig. (8) shows that the effect of the rotor speed on rotor current density. It has the largest value at standstill, with speed increasing the rotor current density decreases and this due to the rotor slip.

It is clear from Figs. $(7,8)$, that the skin effect in disc-rotor conducting sheet does not noticeably influence the rotor current density which is constant along the disc boundary. 
Proceedings of the $5^{\text {th }}$ ICEENG Conference, 16-18 May, 2006

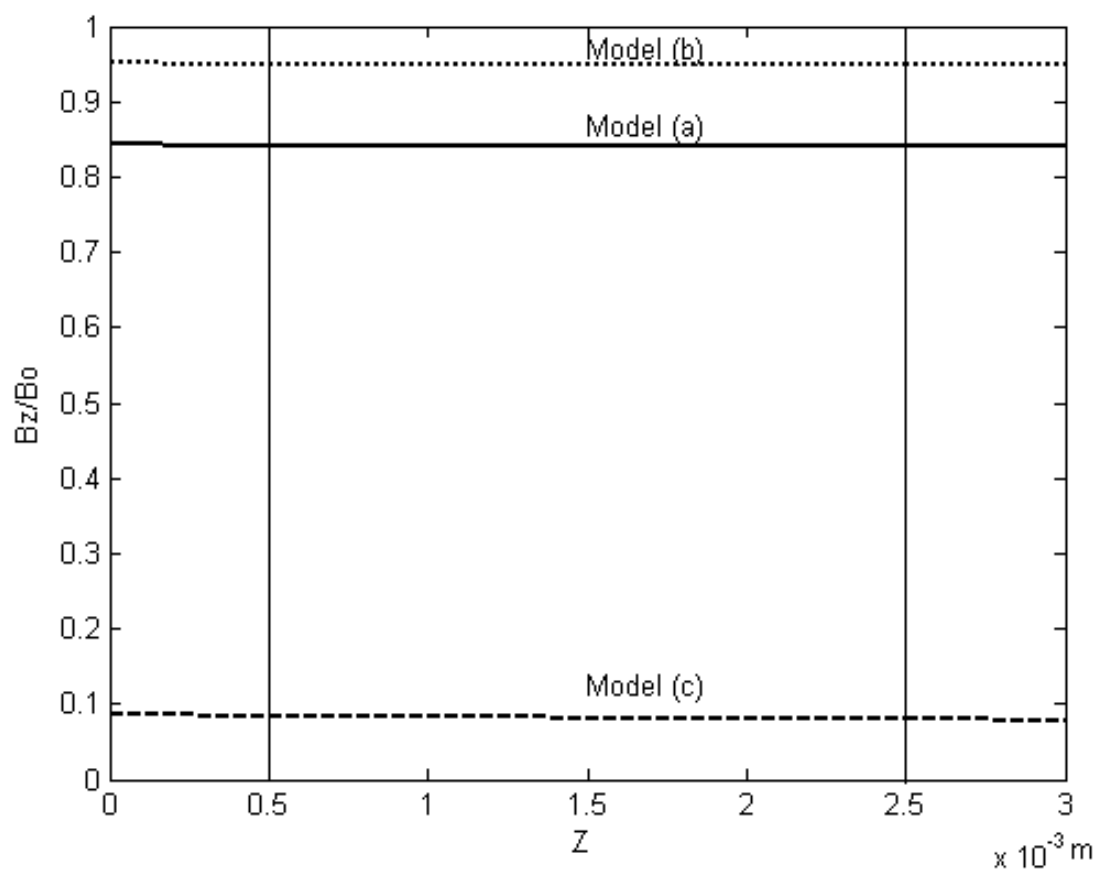

(a)

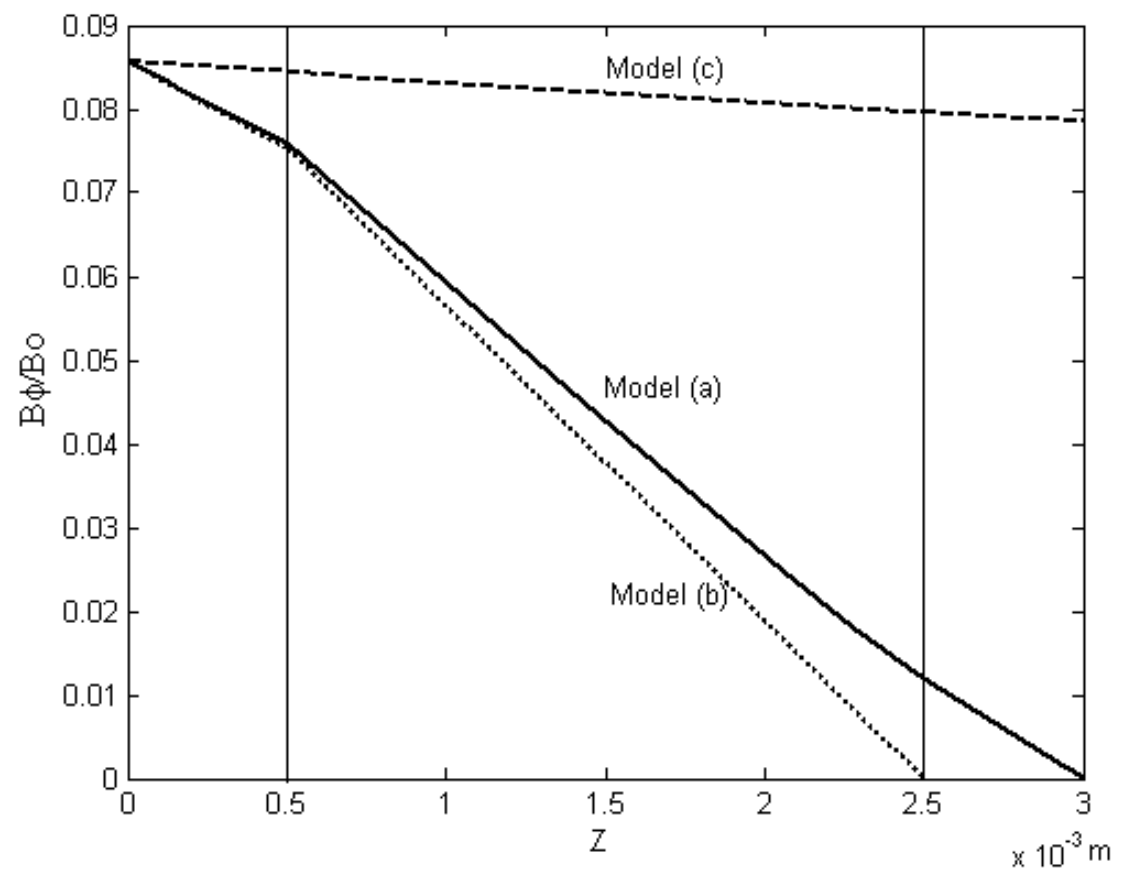

Fig. 4. Distribution of the normalized air-gap flux density for the three model $(a, b, c)$ at constant speed

(a) Axial component

(b) Tangential component 
Proceedings of the $5^{\text {th }}$ ICEENG Conference, 16-18 May, 2006

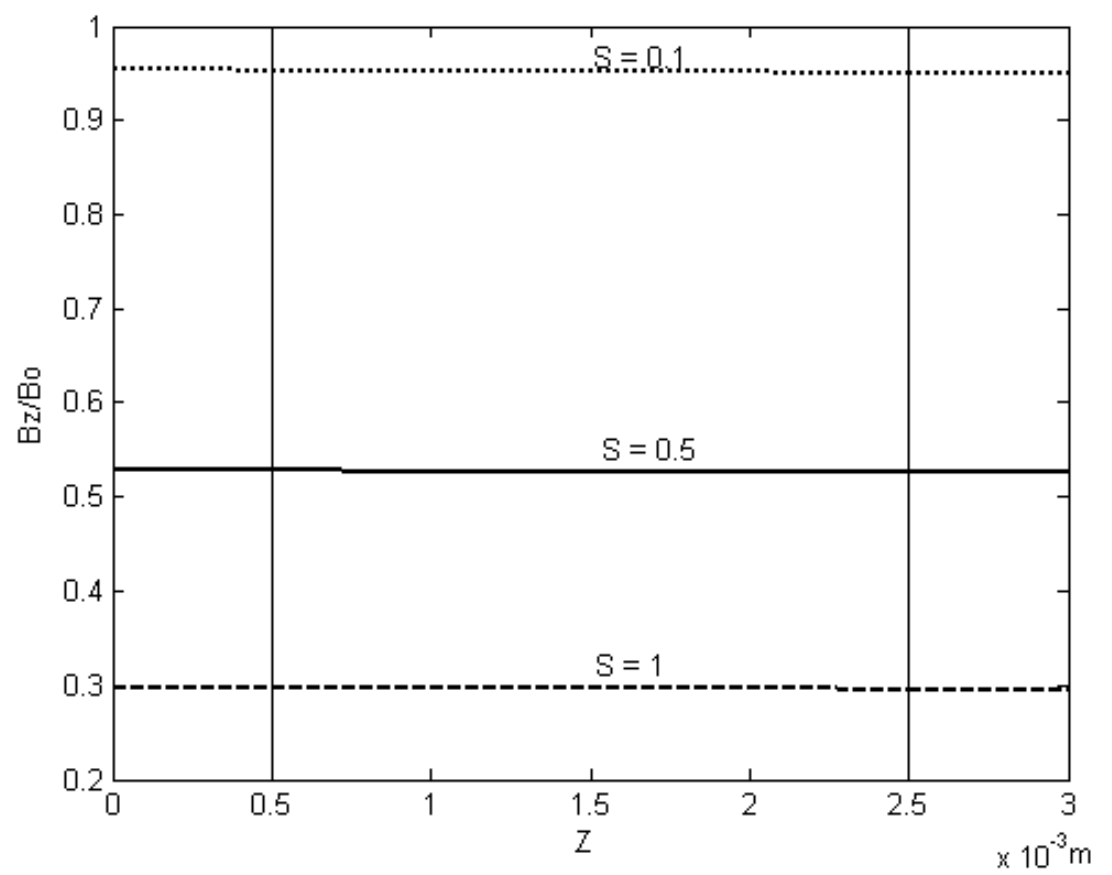

Fig. 5. Distribution of the normalized axial air-gap flux density component for the model (a) at different slips

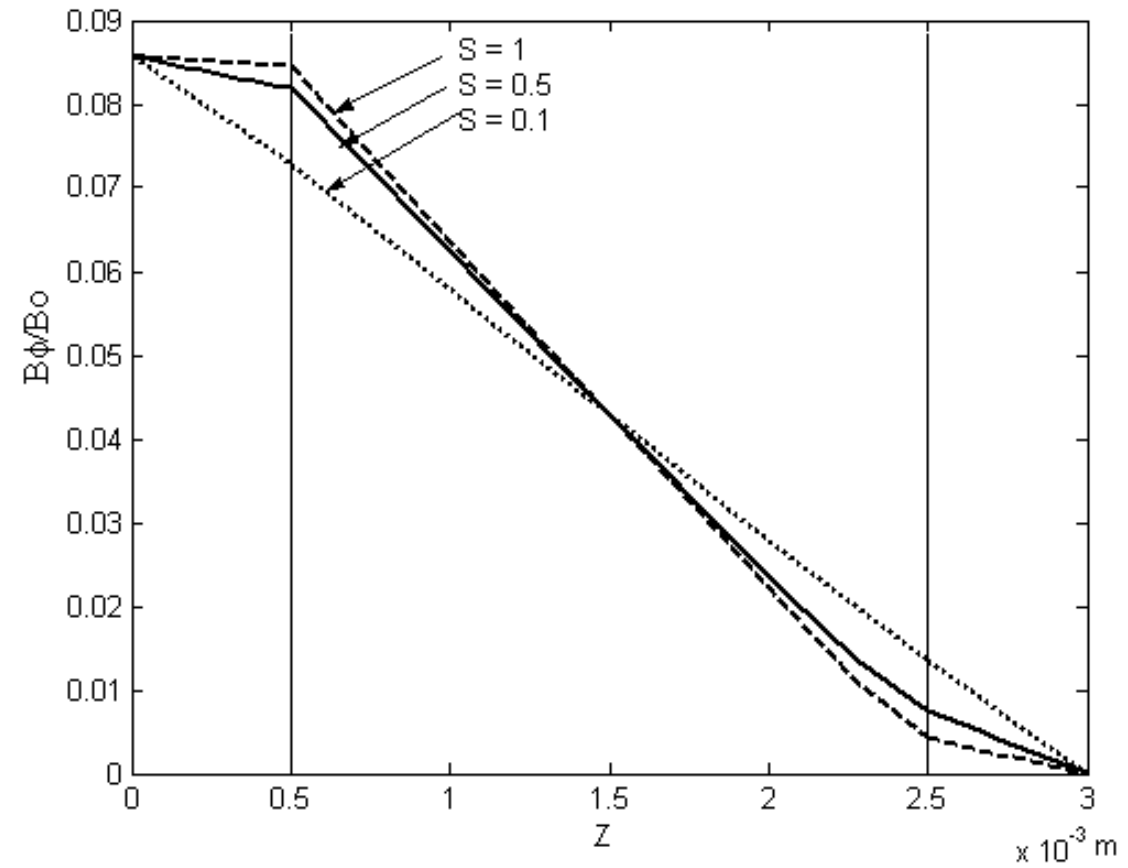

Fig. 6. Distribution of the normalized tangential air-gap flux density component for the model (a) at different slips 
Proceedings of the $5^{\text {th }}$ ICEENG Conference, 16-18 May, 2006

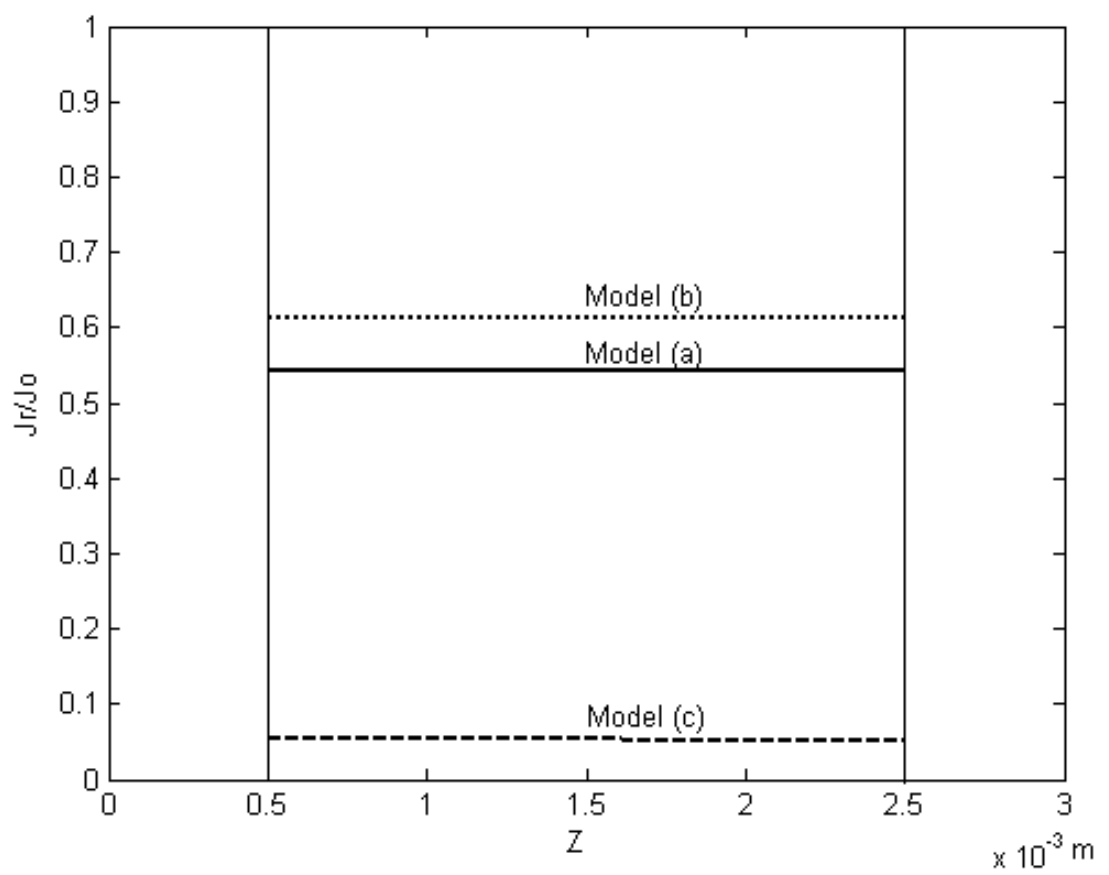

Fig. 7. Normalized rotor current density for the three models $(a, b, c)$ at constant speed

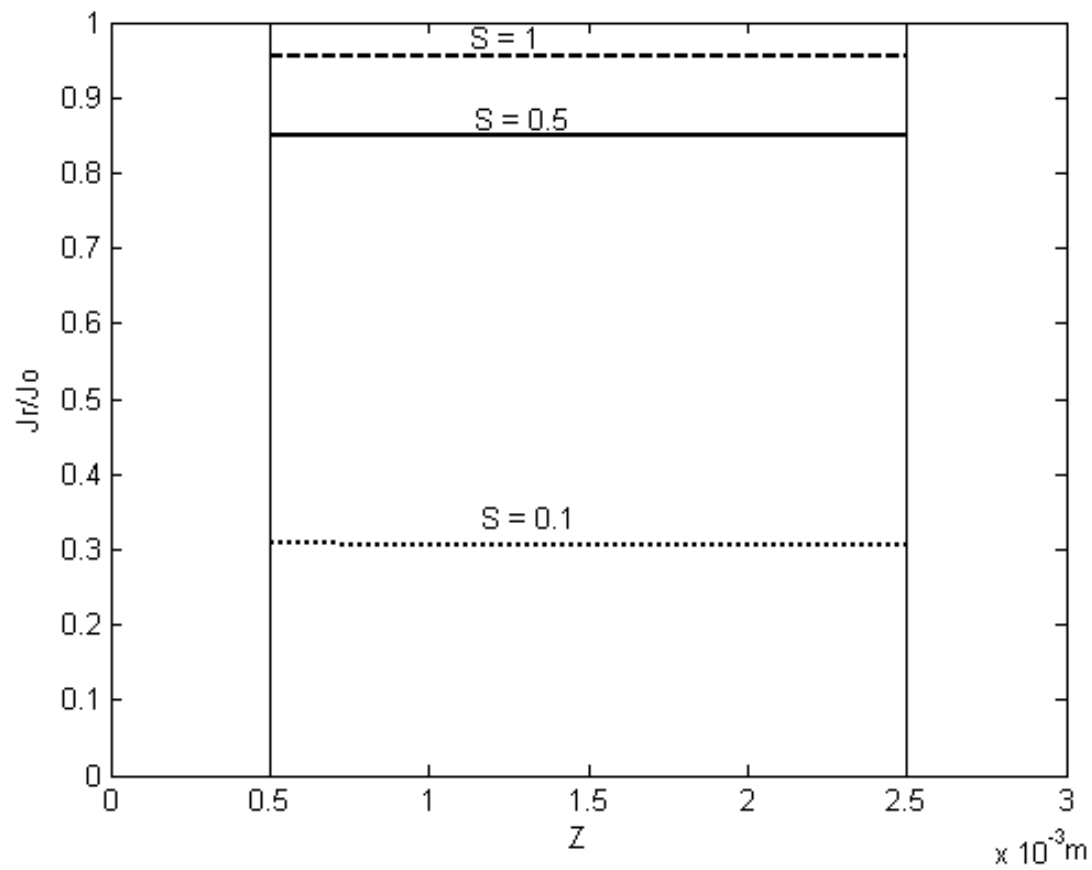

Fig. 8. Normalized rotor current density for the model (a) at different slips 


\section{Air-Gap Impedance Equation}

A simple solution for the disc motor was obtained by integrating the differential equation of a disc-rotor induction motor [1] after it has been adapted to the modified boundary conditions. An adequate representation of the disc motor, which involves only the two air-gap regions, neglecting the disc-rotor thickness is shown in Fig. 3. The vector potential expressions are considerably simplified by assuming rotor surface conductivity $\sigma^{\prime}=\sigma . t_{\mathrm{c}}$. As the skin effect in the disc rotor have no effect on the rotor current density, so can be ignored. The surface conductivity $\sigma$ is the measure for the motor performance. The solution of the magnetic vector potential equations, in the two air-gap regions becomes:

$V_{r 1}=p_{1} \cdot e^{a z}+q_{1} \cdot e^{-a z}, V_{r 2}=p_{2} \cdot e^{a z}+q_{2} \cdot e^{-a z}$

Flux density components $B_{\varphi}$ and $B_{z}$ in the two-regions and the disc-rotor electric loading can be obtained from the relations:

$B_{z n}=j a V_{r n}$

The unknown integrations constants $p$ and $q$ are to be determined by boundary conditions of the problem. At the first air-gap-stator boundary $(z=0)$, the continuity of $H_{\varphi}$ is applied, yielding:

$p_{1}-q_{1}=-\frac{\mu_{0}}{a} A_{s}$

Where $A_{s}$ is the stator- electric loading. At $z=g$, the continuity condition of the normal flux density is applied yielding:

$p_{1} e^{a g}-q_{1} e^{-a g}=p_{2} e^{a g}+q_{2} e^{-a g}$

With $\mathrm{g}=g_{o}+t_{c} / 2$ where $\mathrm{g}_{\mathrm{o}}$ is the mechanical air-gap length and $\mathrm{t}_{\mathrm{c}}$ is the disc-rotor thickness. The disc-rotor electric loading can be expressed in terms of magnetic field intensity to give:

$p_{1}(1+c) e^{a g}-q_{1}(1-c) e^{-a g}=p_{2} e^{a g}+q_{2} e^{-a g}$

with $c=j \frac{\omega \mu \sigma . s}{a}$

at $\mathrm{z}=2 g$, the continuity of $\mathrm{H}_{\vartheta}$ is applied yielding :

$p_{2} e^{2 a g}-q_{2} e^{-2 a g}=0$

The solution of equations (36-40) simultaneously, gives the four integration constants, corresponding to the two regions of the model as:

$p_{1}=\frac{-\mu_{o} A_{s}}{a} \frac{\left[c . e^{-4 a g}+c \cdot e^{-2 a g}-2 . e^{-4 a g}\right]}{\left.2 . c \cdot e^{-2 a g}+c . e^{-4 a g}-2 \cdot e^{-4 a g}+c+2\right]}$ 


$$
\begin{aligned}
& q_{1}=\frac{\mu_{o} A_{s}}{a} \frac{\left[c . e^{-2 a g}+c+2\right]}{\left[2 . c \cdot e^{-2 a g}+c \cdot e^{-4 a g}-2 \cdot e^{-4 a g}+c+2\right]} \\
& p_{2}=\frac{\mu_{o} A_{s}}{a}\left[2 . c . e^{-2 a g}+c \cdot e^{-4 a g}-2 \cdot e^{-4 a g}+c+2\right] \\
& p_{2}=\frac{\mu_{o} A_{s}}{a}\left[2 . c . e^{-2 a g}+c . e^{-4 a g}-2 . e^{-4 a g}+c+2\right]
\end{aligned}
$$

Finally, substitution of the resulting values of $p$ and $q$ into (33 and 34), gives the expression of the normal flux density component as:

$$
B_{z=0}=j \mu_{0} A_{s} \frac{2 \cosh 2 a g+c \sinh 2 a g}{2 \sinh 2 a g+c(1+\cosh 2 a g)}
$$

Once the normal component of the air-gap flux density at the stator surface has been determined, the magnetization voltage induced in each phase is then:

$$
E_{m}=j D \frac{2 \cosh 2 a g+\frac{c}{2} \sinh 2 a g}{2 \sinh 2 a g+\frac{c}{2}(1+\cosh 2 a g)} . I_{s}
$$

where

$$
D=\frac{2 m_{s} \omega \mu_{0}\left(W_{s} K_{w}\right)^{2}\left(R_{o}-R_{i}\right)}{p \pi}
$$

$K_{w}$ is the winding factor, $W_{\mathrm{s}}$ is the number of turns per phase, and $I_{s}$ is the r.m.s. value of the stator current. In terms of the air-gap impedance $Z_{g}$, the stator emf is:

$E_{m}=Z_{g} . I_{s}$

With $Z_{\mathrm{g}}$ represents the combination of the magnetization reactance and the equivalent rotor impedance.

Hence, the air-gap impedance expression with respect to the first model of Fig. $2 \mathrm{a}$ is then:

$$
Z_{g a}=j D \frac{2 \cosh 2 a g+\frac{c}{2} \sinh 2 a g}{2 \sinh 2 a g+\frac{c}{2}(1+\cosh 2 a g)}
$$

The air-gap impedances of the two other models can be obtained from Eq. (48) with the following assumptions:

1-The motor corresponding to model $2 \mathrm{~b}$, has a single sided stator with an iron backing rotor, the outer air-gap length is zero while $2 g$ is replaced by $g^{\prime}=g_{o}+t_{c}$, the airgap impedance is then :

$$
Z_{g b}=j D \frac{\cosh a g^{\prime}+c \cdot \sinh a g^{\prime}}{\sinh a g^{\prime}+c \cdot \cosh a g^{\prime}}
$$


2- the motor of model 2c, has a single-sided stator with non-ferromagnetic disc-rotor, the field in the outer air-gap region is modified, while the inner air-gap length is still $\mathrm{g}$, the air-gap impedance is then:

$$
Z_{g c}=j D \frac{e^{a g^{\prime}}+\frac{c}{2} \cdot \sinh a g}{e^{a g^{\prime}}+\frac{c}{2} \cdot \cosh a g}
$$

\section{Separation of Individual Parameters}

The individual equivalent circuit parameters may be defined in different ways depending on the form used in writing the air-gap impedance [2]. The equivalent circuit of an induction motor is proposed here to represent the magnetization reactance $X_{m}$ in parallel with the disc rotor impedance (resistance $R_{r}$ and leakage reactance $X_{r}$ ). Therefore. $Z_{\mathrm{g}}$ can be written as:

$$
Z_{g}=j X_{m} \frac{R_{r}+j s X_{r}}{R_{r}+j s\left(X_{m}+X_{r}\right)}
$$

Both numerator and denominator of Eq. (48) are extended by the same factor $2 D / c_{o}$ with a real constant $\mathrm{c}_{\mathrm{o}}=c / j s$. The resulting impedance equation is arranged in a form suitable for comparison with that given from the equivalent circuit.

The individual parameters of the disc-rotor equivalent circuit, corresponding to the model of Fig. la are :

$$
X_{m a}=D \cdot \operatorname{coth} 2 a g, X_{r a}=X_{m a} \frac{\sinh ^{2} 2 a g}{1+\cosh 2 a g}, R_{r a}=X_{m a} \frac{\sinh 4 a g}{c_{o}(1+\cosh 2 a g)}
$$

Following the same procedure used in separating the equivalent circuit parameters of the first disc motor model $2 \mathrm{a}$, the other two models $2 \mathrm{~b}$ and $2 \mathrm{c}$ can be obtained similarly as:

$$
X_{m b}=D \cdot \operatorname{coth} 2 a g^{`}, X_{r b}=X_{m b} \cdot \sinh ^{2} 2 a g^{`}, R_{r b}=X_{m b} \frac{\sinh a g^{`} \cdot \cosh a g^{`}}{c_{o}} \quad(53 \mathrm{a}, \mathrm{b}, \mathrm{c})
$$

and

$$
X_{m c}=D, X_{r c}=X_{m c} \frac{e^{2 a g^{\prime}}-1}{2}, R_{r c}=X_{m c} \frac{e^{2 a g^{\prime}}}{c_{o}\left(\cosh a g^{\prime}-\sinh a g^{\prime}\right)}
$$

The two-phase disc-rotor induction motor can be represent by $\Gamma$-type equivalent circuit of unbalanced two-phase motor [5]. 


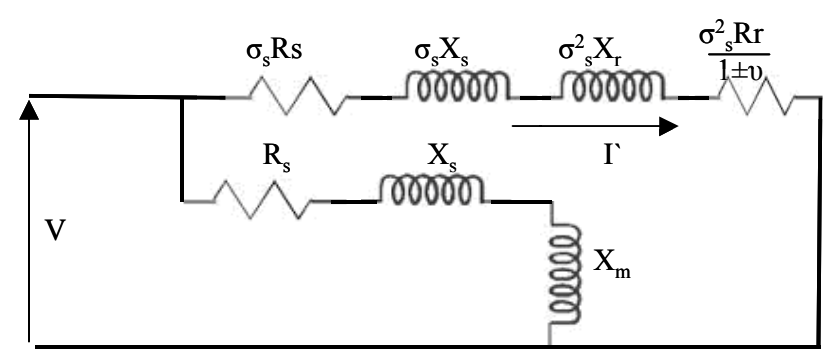

Fig.9. $\Gamma$-type equivalent circuit of on phase of a two-phase disc-rotor induction motor

Where

$$
\sigma_{s}=1+\frac{X_{s}}{X_{m}}-j \frac{R_{s}}{X_{m}} \quad, v=\frac{n}{n_{s}}
$$

Since the actual disc-rotor is replaced in the used models by an infinitely thin current sheet, then the equivalent circuit parameters are independent on the speed. As shown from Fig. $(7,8)$, the rotor current density is constant along the disc boundary, this attributed to the skin effect in disc-rotor conducting sheet does not noticeably influence the rotor current density, so it have no effect on the rotor equivalent resistance . The equivalent circuit parameters of the disc motors, corresponding to their design data, are computed and tabulated for comparison in table 1.

Some of the specific data pertained to the disc motor described in this paper are:$\mathrm{m}_{\mathrm{s}}=2, \mathrm{~W}_{\mathrm{s}}=850$ turns, $\sigma_{\mathrm{s}}=2 * 10^{7} \mathrm{~S} / \mathrm{m}, 2 \mathrm{P}=4$ poles, $\mathrm{f}=50 \mathrm{~Hz}, \mathrm{R}_{\mathrm{o}}=9 \mathrm{~cm}, \mathrm{R}_{\mathrm{i}}=5 \mathrm{~cm}$, $\mathrm{t}_{\mathrm{c}}=2 \mathrm{~mm}$ (Aluminum), $\mathrm{g}_{\mathrm{o}}=0.5 \mathrm{~mm}$.

\section{Table1:}

The individual parameters of the disc motor corresponding to the models $\mathrm{a}, \mathrm{b}$ and $\mathrm{c}$

\begin{tabular}{|c|c|c|c|c|c|c|c|c|c|c|c|}
\hline \multicolumn{3}{|c|}{$\begin{array}{c}\text { Parameters } \\
\text { Design Data }\end{array}$} & \multicolumn{3}{|c|}{$\mathrm{X}_{\mathrm{m}}(\Omega)$} & \multicolumn{3}{|c|}{$\mathrm{X}_{\mathrm{r}}(\Omega)$} & \multicolumn{3}{|c|}{$\mathrm{R}_{\mathrm{r}}(\Omega)$} \\
\hline $\mathrm{g}(\mathrm{mm})$ & $\mathrm{t}_{\mathrm{c}}(\mathrm{mm})$ & $\sigma(\mathrm{s})$ & (a) & (b) & (c) & (a) & (b) & (c) & (a) & (b) & (c) \\
\hline 0.75 & 2 & $2 * 10^{7}$ & $\wedge \wedge, 0$ & TYY & $1 V, 0$ & 1,1 & $1, \varepsilon$ & 1,0 & TY,T & $\Gamma, \Lambda$ & $\varepsilon$ \\
\hline 0.50 & 2 & $2 * 10^{7}$ & $1 \cdot r, q$ & $r \leq 0$ & $1 V, 0$ & 1,0 & $1, r$ & $1, r$ & rY,r & $\Gamma, \wedge$ & $r q, r$ \\
\hline 0.25 & 2 & $2 * 10^{7}$ & Irr & TVY & $1 V, 0$ & $1, r$ & $1,1 Y$ & $1, r$ & $r r$ & $r, V$ & $\Gamma \wedge, r$ \\
\hline 0.50 & 1.5 & $2 * 10^{7}$ & $17 \varepsilon$ & $\varepsilon \cdot \lambda$ & $r r, r$ & $1, V$ & $1, r$ & $1, \varepsilon$ & ov & $07, \varepsilon$ & $77, V$ \\
\hline 0.50 & 1 & $2 * 10^{7}$ & $r \cdot V$ & 117 & ro & $r$ & 1,0 & 1,7 & $I Y V, V$ & $\mid r T, 7$ & $1 \leq \varepsilon$ \\
\hline 0.50 & 2 & $10^{7}$ & $r \cdot T$ & $\varepsilon 9$. & ro & $r$ & $Y, 0$ & $Y, V$ & 119 & ITV & 107 \\
\hline 0.50 & 2 & $4 * 10^{7}$ & $01, \varepsilon$ & TYY & $\Lambda, \vee$ & $\cdot, 1 / 7$ & $\cdot, 7 \pi$ & $\cdot, \mathrm{TV}$ & $\Lambda, 1$ & $\wedge$ & 9,1 \\
\hline
\end{tabular}




\section{5-Conclusions}

The three constructions for the disc-rotor IM are proposed and their models are considered taking into account the two air-gaps and the rotor thickness. The analysis is considerably simplified by assuming thin rotor with finite surface conductivity $\left(\sigma^{\prime}=\sigma \mathrm{t}_{\mathrm{c}}\right)$. Axial and tangential components of the air-gap flux density and radial rotor current density for the three models $(a, b, c)$ of the disc-motor are distributed. This analysis enables disc-rotor induction motor characteristics to be obtained with very simple steps of calculations. A suitable operating condition may be realized for a motor of single sided stator with an iron backing disc-rotor (model b). The equivalent circuit individual parameters for each model are obtained directly from the magnetic vector potential which is readily adaptable to the evaluation of the air-gap impedance. A comparative study on the effect of the main design data on the disc motor equivalent circuit parameters are investigated. This method of analysis investigates the machine parameters with suitable accuracy and with simple calculations of magnetic flux at each region of the disc-rotor induction motor. Also, this proposed method has the ability of accurate performance prediction for the disc-rotor induction motors, which enables the designer to choose the suitable data for the rotor disc.

\section{6-References}

[1]. S. E. Abdollahi, M.Mirsalim, M. Mirzayee, A. Vahedi, "Performance Analysis of a Solid Rotor Disk Induction Motor", ICEM 2004, Cracowa, Poland, sept. 2004

[2]. A. B, Kotb: "Disc-rotor induction motor with mechanical speed-control", Sci. Boll. Fac. Eng. Ain Shams Univ,, No. 4, Vol. 31. Part II, p. 420, 1996.

[3]. Hosseini Aliabadi, A.Vahedi and S. E. Abdollahi," Investigation of Wedge Holders Effect on Torque Calculation of a Solid Rotor disc Induction Motor using Two-Dimensional Finite Elements Method M", IEEE GCC, Maname, Bahrain, November 2004

[4]. M. Shalaby: "Phase-angle control of the repulsion/drive force ratio in twophase linear induction motor with non-ferromagnetic secondary", IEE Proceedings, Vol. 132, p. 81, 1986.

[5]. M, Saleh and M. A. Shehata: "Effect of the method of control on the transfer function parameters of a two-phase servomotor". Measurement and control. Vol. 6.p.517,1973.

[6].Weh, Mosebach and May; "Analysis and characteristics of the disk-rotor IM", Electric machines and electromechanics Vol. 1, p. 87,1976. 